Cómo citar este trabajo: Barros Pozo, P. M., \& Martin-Vide, J. (2018). Influencia térmica antrópica local y global en el observatorio Fabra para el periodo 1924-2016. Boletín de la Asociación de Geógrafos Españoles, 79, 2515, 1-23. http://dx.doi.org/10.21138/bage.2515a

\title{
Influencia térmica antrópica local y global en el observatorio Fabra para el periodo 1924-2016
}

Local and global anthropic thermal influence at Fabra observatory for the period 1924-2016

\author{
Patricia Magaly Barros Pozo \\ patba_86@hotmail.com \\ Facultad de Geografía e Historia \\ Universidad de Barcelona (España) \\ Javier Martin-Vide \\ jmartinvide@ub.edu \\ Grupo de Climatología \\ Universidad de Barcelona (España)
}

\section{Resumen}

Se presenta una primera aproximación en la diferenciación de la contribución local y global en el incremento de la temperatura del aire en Barcelona. Para ello, se busca determinar la influencia local del área metropolitana de Barcelona en el aumento de la temperatura del aire registrado en el observatorio Fabra, para el periodo 1924-2016, a diferencia del observatorio del Ebro (observatorio de referencia) que presenta solamente la influencia del calentamiento global por ubicarse en una zona rural. La temperatura registrada en el observatorio Fabra presentó una tendencia significativa al incremento durante 1924-2016, incrementándose la temperatura media anual en $1,26{ }^{\circ} \mathrm{C}$. Durante el subperiodo $1972-2016$, la temperatura registró una tasa más acelerada en comparación con el subperiodo 1924-1971, coincidiendo el primer subperiodo con los años en que las investigaciones sobre el cambio climático muestran resultados más concluyentes 
de la influencia antropogénica en el aumento de la temperatura del aire. Considerando dicho subperiodo, el observatorio Fabra registró una influencia térmica local del área metropolitana de Barcelona de $0,0073{ }^{\circ} \mathrm{C} /$ año en la temperatura media anual, mientras que $0,0386{ }^{\circ} \mathrm{C} /$ año correspondió al aporte del calentamiento global, registrado en el observatorio del Ebro.

Palabras clave: área metropolitana de Barcelona; observatorio Fabra; observatorio del Ebro; temperatura; calentamiento global.

\begin{abstract}
This article shows a first approach in the differentiation between the local and global contribution in the increase of the air temperature in Barcelona. Accordingly, the local influence of the Barcelona metropolitan area on the increase of air temperature is assessed by the recorded data at the Fabra observatory for the period 1924-2016; and then, it is compared with the Ebro observatory's (reference observatory), which only presents a global warming influence as it is located in a rural zone.
\end{abstract}

The results show that the temperature recorded in the Fabra observatory has a significant increasing trend; in the term 1924-2016, where the increase of the average monthly temperature was identified in $1,26{ }^{\circ} \mathrm{C}$. The increasing temperature rate recorded for the subperiod $1972-2016$ in the Fabra observatory was faster than the one registered for the subperiod 1924-1971, coinciding with the years in which climate change researches have shown more conclusive results of the anthropogenic influence in the increase of air temperature. In this subperiod, the results indicate that the Fabra observatory registered a local thermal influence of the Barcelona metropolitan area which contributes in an increase of $0,0073{ }^{\circ} \mathrm{C} /$ year in the average annual temperature, whereas the contribution of the global warming, registered at Ebro observatory, corresponded to an increase of $0,0386^{\circ} \mathrm{C} /$ year.

Key words: Barcelona metropolitan area; Fabra observatory; Ebro observatory; temperature; global warming.

\title{
1 Introducción
}

La interacción del ser humano con su ambiente origina diversas alteraciones en el medio que pueden ser calificadas como positivas o negativas. Como resultado de esta interacción, el hombre ejerce influencia en el sistema climático a diferentes escalas, desde la local hasta la global, por lo que su inclusión como parte del sistema climático es necesaria. Es así, que se considera al subsistema socioeconómico como el sexto subsistema del sistema climático (Martín Vide, 2008). 
El crecimiento desmedido de la población mundial ${ }^{1}$ ha originado que los impactos ocasionados al medio por las actividades antropógenas sean predominantemente negativos, de mayor significación y de mayor amplitud espacial. Se ha evidenciado que a la variabilidad climática natural se añade ya una incidencia antrópica en el sistema climático, tal como lo indica el quinto informe del Grupo Intergubernamental de Expertos sobre el Cambio Climático (IPCC): "La influencia humana en el sistema climático es clara, y las emisiones antropógenas recientes de gases de efecto invernadero son las más altas de la historia. Los cambios climáticos recientes han tenido impactos generalizados en los sistemas humanos y naturales" (Grupo Intergubernamental de Expertos sobre el Cambio Climático, 2015).

La temperatura media global del aire se ha incrementado, durante los últimos 100 años, debido principalmente a las emisiones gaseosas de efecto invernadero, alcanzándose concentraciones de dióxido de carbono $\left(\mathrm{CO}_{2}\right)$, metano $\left(\mathrm{CH}_{4}\right)$ y óxido nitroso $\left(\mathrm{N}_{2} \mathrm{O}\right)$ que no se han evidenciado en los últimos 800000 años y triplicándose, desde 1970, las emisiones de $\mathrm{CO}_{2}$ provenientes de la combustión de materiales fósiles y de procesos industriales, responsables alrededor del $78 \%$ del incremento de los gases de efecto invernadero en la atmósfera (Grupo Intergubernamental de Expertos sobre el Cambio Climático, 2015). Especificamente en España, las temperaturas registradas durante el siglo XX, especialmente a partir de los años 70, evidencian el incremento sostenido de la temperatura del aire, mostrando una tendencia superior a la observada a nivel global, habiéndose registrado un incremento de aproximadamente $0,6{ }^{\circ} \mathrm{C}$ por década en las temperaturas máximas y mínimas (Castro, Martín Vide, y Alonso, 2005). Asimismo, las proyecciones realizadas, para el siglo XXI, evidencian una tendencia progresiva del incremento de la temperatura media, especialmente durante los meses de verano y en las zonas del interior de la Península Ibérica, con mayor frecuencia de días de temperaturas máximas extremas en verano y con una disminución de la precipitación (Moreno Rodríguez, 2005)

Debido al incremento de la temperatura, por actividades humanas, las otras variables climáticas, como la precipitación, se han alterado modificando el clima a escala global ${ }^{3}$ (Martín Vide, 2008). Asimismo, cabe indicar que, también es de esperar que las actividades antropógenas ejerzan

1 La población mundial ha pasado de ser 461 millones, en 1500, (Vallin, 1993, mencionado por Alcañiz, 2008) a ser 7433 millones en 2016 (Fondo de Población de las Naciones Unidas, 2016), aumentando, en 517 años, 16 veces su valor.

2 Órgano intergubernamental, establecido conjuntamente por la Organización Meteorológica Mundial (OMM) y el Programa de las Naciones Unidas para el Medio Ambiente (PNUMA), encargado de realizar evaluaciones científicas y técnicas relacionadas con el cambio climático, proporcionando a los responsables de la formulación de políticas las bases científicas del cambio climático, impactos, riesgos y opciones de adaptación y mitigación (Grupo Intergubernamental de Expertos sobre el Cambio Climático, 2015).

3 El cambio climático, de origen antropógeno, es la alteración del comportamiento de las variables climáticas, por la variación de la composición atmosférica, registrándose modificaciones estadísticamente significativas de estas variables con respecto a su media, las cuales persisten por un periodo prolongado de tiempo (World Meteorological Organization, n.d.). 
influencia en el clima a escala local, presentando su máxima expresión en las ciudades. Las áreas urbanas $^{4}$ se diferencian de su entorno, predominantemente natural y/o rural, principalmente por la sustitución de la superficie del suelo por materiales de construcción antropógenos, por la modificación del relieve y por el número de personas que las habita, ocasionando la fragmentación de los ecosistemas, la alteración del ciclo hidrológico y de la transferencia de materia y energía. Por ello, las ciudades, al ser dinámicas y al encontrarse en continuo crecimiento a nivel horizontal y/o vertical, originan impactos en los componentes ambientales como la atmósfera, modificando los elementos climáticos (temperatura, viento, evaporación, factor de visión del cielo, etc.), dando lugar a un clima urbano que se caracteriza por presentar una temperatura más elevada que su entorno. ${ }^{5}$ La diferencia positiva de la temperatura en las ciudades, especialmente durante las noches (ver Figura 1), con respecto al área que la rodea, da origen al fenómeno denominado isla de calor urbano, el cual en Barcelona presenta mayor intensidad en invierno y en situaciones de calma anticiclónica (Moreno García, 2010). La formación de este fenómeno depende de factores geográficos (p. ej. latitud, altitud), factores urbanos (p. ej. altura de edificios, tipo de materiales de edificaciones) y de los patrones de clima local (U.S. EPA, 2008).

Figura 1. Variación horaria de la temperatura del aire y de la intensidad de la isla de calor urbana

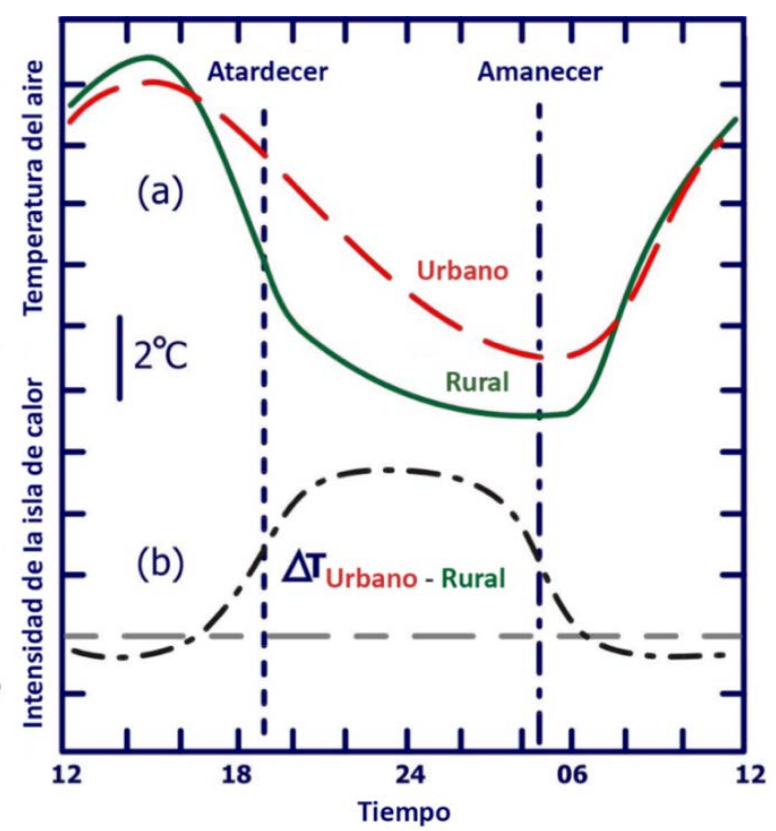

Fuente: U.S. EPA (2008), modificado de Oke (1982) y Runnalls \& Oke (2000)

4 Las áreas urbanas en 1960 concentraban al 33,6\% de la población mundial, mientras que en 2015 acogían al 53,9 \% (Organización de las Naciones Unidas, 2016).

5 Se registran mayores temperaturas en las ciudades debido a la liberación de calor proveniente de procesos de combustión y a la presencia de materiales antropógenos que difieren del suelo y/o vegetación natural en el almacenamiento de calor, en la capacidad de absorción de la radiación solar, en la evaporación, filtración, escurrimiento y almacenamiento de agua (Henry \& Heinke, 1999). 
El área metropolitana de Barcelona, que posee un clima mediterráneo, ha presentado un incremento de la temperatura de $0,07^{\circ} \mathrm{C}$ por decenio, para el periodo de 1780 a 2014, lo que corresponde a $1,6^{\circ} \mathrm{C}$ más que el siglo XVIII, y sólo considerando el periodo de 1950 a 2014, el incremento fue de $0,23^{\circ} \mathrm{C}$ por decenio (Institut d'Estudis Catalans y Generalitat de Catalunya, 2016). En la Figura 2, se aprecia que la temperatura media anual del aire en Barcelona ha mostrado un crecimiento desde el comienzo del siglo XX, registrándose a partir de mediados de los años 70 un incremento más acelerado, lo que coincide con el incremento a nivel mundial de las concentraciones de gases de efecto invernadero en la atmósfera. Por otra parte, las proyecciones climáticas realizadas para Cataluña indican que el incremento de la temperatura podría ser entre 0,3 y 1,0 ${ }^{\circ} \mathrm{C}$ para los años 2012 al 2021, y entre 0,9 y 1,9 ${ }^{\circ} \mathrm{C}$ para el periodo 2031 al 2050, aumentando el número de noches tropicales $\left(T n \geq 20^{\circ} \mathrm{C}\right.$ ) en la zona litoral y pre-litoral (Institut d'Estudis Catalans y Generalitat de Catalunya, 2016). Este incremento de temperatura, asociado al cambio climático, podría acentuar los efectos negativos de la isla de calor.

\section{Figura 2. Evolución temporal de la temperatura media anual de Barcelona (1780 a 2016)}

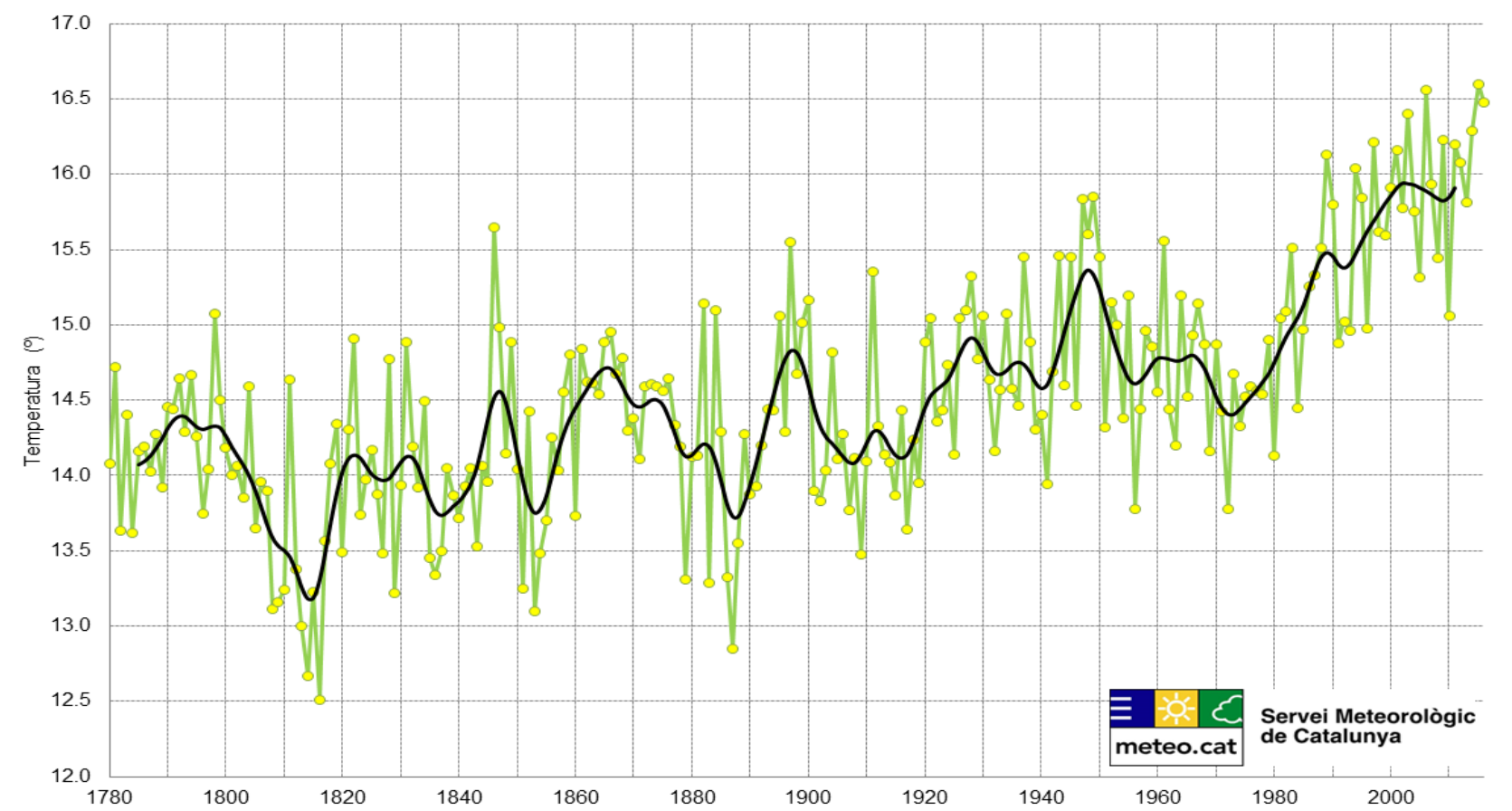

Fuente: Institut d'Estudis Catalans y Generalitat de Catalunya (2016)

La presente investigación busca aportar, a los estudios ya realizados sobre la isla de calor urbano en Barcelona y sobre el cambio climático, información sobre la contribución que ejerce el área metropolitana de Barcelona en el incremento de la temperatura del aire entre los años 1924 y 2016. Se han realizado estudios como el de Martín Vide et al. (2016), en el que se ha determinado que para el periodo 1914 al 2014, se ha incrementado en un 1,3 ${ }^{\circ} \mathrm{C}$ la temperatura media del aire en el observatorio Fabra, debido al cambio climático antropógeno, sin hablarse de la 
influencia urbana existente al encontrarse el observatorio Fabra en los límites de la ciudad de Barcelona, e implícitamente eliminada en el análisis. En tal sentido, el objetivo principal de la investigación es determinar la influencia local que ejerce el área metropolitana de Barcelona en el incremento de la temperatura del aire en el observatorio Fabra para el periodo de 1924 a 2016.

Cabe resaltar que existen trabajos con un objetivo similar al del presente artículo, es decir, el análisis de la señal urbana en la evolución de la temperatura en el contexto del calentamiento global. Destacan entre ellos los de Hamdi et al. (2009) para el caso de Bruselas y Jones y Lister (2009) para Londres. En general, se concluye en la importante huella que dejan las islas de calor en las series de temperatura de las ciudades, con un incremento térmico a la par del crecimiento urbano (Hamdi \& Van de Vyver, 2011). Diversos estudios enfatizan el aumento de la intensidad de la isla de calor y sus efectos con el calentamiento global, en la actualidad o en el futuro, como en el caso de Glasglow (Emmanuel \& Krüger, 2012) o en el de Melbourne (Sachindra et al., 2016). Mediante modelización climática, en alguna investigación, se concluye que con el cambio climático no aumentará la intensidad de la isla de calor, aunque sí sus efectos negativos, como en Bruselas y París (Hamdi et al., 2015). Y un amplio conjunto de trabajos tratan aspectos económicos, de confort térmico y necesidades de refrigeración, y de ecología en las ciudades bajo el cambio climático (Grimm et al., 2008; Hwang, et al., 2017; Estrada et al., 2017).

\section{2 Área de estudio}

El área de estudio se limita al área metropolitana de Barcelona que se encuentra localizado al noreste de España, en la provincia de Barcelona, en la Comunidad Autónoma de Cataluña. Se ubica en la zona litoral y prelitoral de Cataluña, drenada por los ríos Besòs y Llobregat, que dividen el territorio hasta su desembocadura en el mar Mediterráneo y dan lugar a llanuras aluviales, así como al del río Llobregat. Los elementos orográficos más destacables son la sierra de Collserola, el macizo del Garraf y la sierra de la Marina, que han condicionado y condicionan la ocupación del suelo. De acuerdo a ello, en las zonas planas y con baja pendiente predominan los centros urbanos, en la llanura aluvial y en el delta del río Llobregat se encuentran los campos agrícolas, mientras que los espacios con pendientes más elevadas se caracterizan por la presencia de áreas forestales (Àrea Metropolitana de Barcelona \& Barcelona Regional, 2014). La Figura 3 muestra la ubicación del área metropolitana de Barcelona.

El área metropolitana de Barcelona, reconocido oficialmente por la Ley 31/2010, se encuentra conformada por la ciudad de Barcelona y por los 35 municipios que la rodean. Presenta una extensión de 633 km2, lo que representa el 2 \% del territorio de la Comunidad Autónoma de Cataluña, y posee una población de 3226600 habitantes, de acuerdo al Institut d'Estadística de Catalunya (2016), es decir el equivalente al 42,9\% de la población total de Cataluña, presentando 
una alta densidad poblacional equivalente a 5097 habitantes $/ \mathrm{km}^{2}$. En la Tabla 1 se indican los municipios que son considerados como parte del área metropolitana de Barcelona.

\section{Figura 3. Ubicación del área metropolitana de Barcelona}

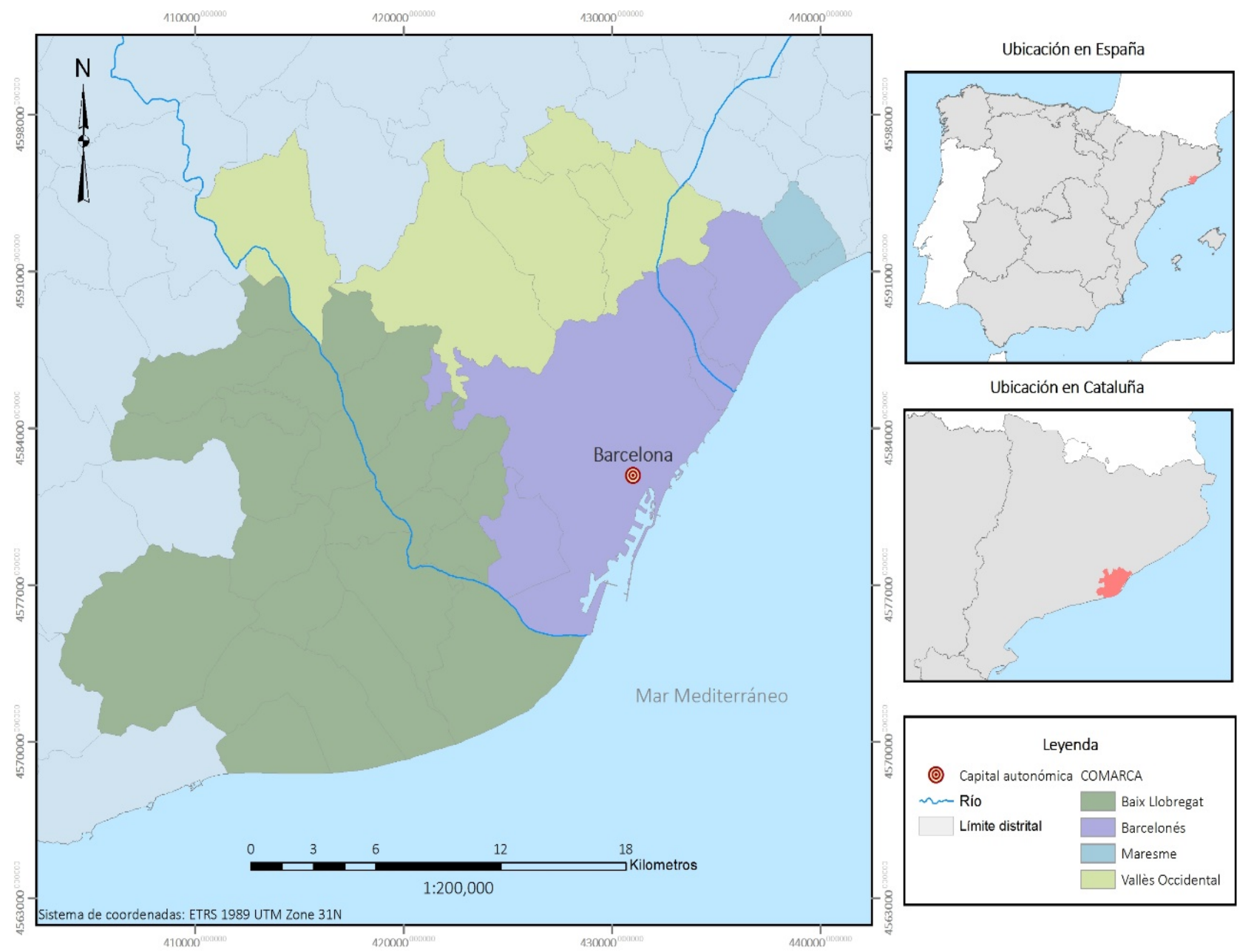

Fuente: elaboración propia

Según Ferrer i Aixalà (1996), a mediados del siglo XIX, Barcelona incrementó su población rapidamente por el auge de la industrialización de la ciudad, duplicando su número de habitantes entre los años 1900 y 1950, inicialmente debido a la inmigración de personas foráneas provenientes de las zonas rurales de Cataluña y posteriormente, en los años 1920, por la llegada de personas provenientes del exterior de la región. Este gran flujo migratorio contribuyó a la expansión horizontal de las áreas urbanas en el territorio del área metropolitana de Barcelona, creándose nuevas viviendas y produciendo el crecimiento de núcleos urbanos periféricos, originándose así la transformación física del territorio, la modificación del suelo no urbano en suelo urbano. 
Tabla 1. Distritos que conforman el área metropolitana de Barcelona

\begin{tabular}{|l|ll|}
\hline \multicolumn{1}{|c|}{ Comarca } & \multicolumn{1}{c|}{ Municipios } \\
\hline \multirow{3}{*}{ Barcelonès } & Barcelona & Hospitalet de Llobregat \\
& Badalona & Sant Adrià de Besòs \\
& Santa Coloma de Gramenet \\
\hline \multirow{4}{*}{ Baix Llobregat } & Begues & El Prat de Llobregat \\
& Castelldefels & Sant Andreu de la Barca \\
& Cervelló & Sant Boi de Llobregat \\
& Corbera de Llobregat & Sant Climent de Llobregat \\
& Cornellà de Llobregat & Sant Feliu de Llobregat \\
& Esplugues de Llobregat & Sant Joan Despí \\
& Gavà & Sant Just Desvern \\
& Molins de Rei, & Sant Vicenç dels Horts \\
& Pallejà & Santa Coloma de Cervelló \\
& La Palma de Cervelló & Torrelles de Llobregat \\
& El Papiol & Viladecans \\
\hline \multirow{3}{*}{ Vallès Occidental } & Badia del Vallès & Cerdanyola del Vallès \\
& Barberà del Vallès & Montcada i Reixac \\
& Castellbisbal & Ripollet \\
\hline Maresme & Sant Cugat del Vallès \\
\hline & Montgat & Tiana \\
\hline
\end{tabular}

Fuente: Área Metropolitana de Barcelona \& Barcelona Regional (2014)

\section{Metodología y datos}

Para el análisis de la evolución de la temperatura del aire durante los años 1924 a 2016, se emplearon los datos de los promedios mensuales de la temperatura máxima, media y mínima de los observatorios Fabra y del Ebro correspondientes a un periodo de 93 años de análisis. Estos datos, de excelente calidad, se obtuvieron a partir de fuentes oficiales, la Real Academia de Ciencias y Artes de Barcelona (RACAB), propiedad del observatorio barcelonés, y la Agencia Estatal de Meteorología (AEMET), a cuya red de observación pertenece el observatorio del Ebro. Estos datos sin lagunas significantes, homogéneos y de gran calidad, fueron sometidos a un control de calidad que evidenció la garantía de homogeneidad que las citadas instituciones certifican.

Hay que indicar que la data meteorológica del observatorio del Ebro presentó algunos vacíos de información, 15 datos, los cuales correspondieron a un año completo (1938) y a datos de diciembre de 1924, julio de 1934 y enero de 1936. Sin embargo, se considera que esta ausencia de datos no altera la tendencia de la temperatura para el periodo estudiado, ya que solo representan el 1,3\% del total analizado.

La Figura 4 muestra la ubicación de los dos observatorios analizados. Fabra se localiza en el municipio de Barcelona, en las coordenadas geográficas $41^{\circ} 25,100^{\prime} \mathrm{N}$ y $2^{\circ} 07,450^{\prime} \mathrm{E}$, específicamente en el distrito Sarriá-San Gervasio, comarca del Barcelonès, provincia de Barcelona, 
a una altitud de $415 \mathrm{msnm}$. El observatorio del Ebro se encuentra localizado en las coordenadas $40^{\circ} 49,261^{\prime} \mathrm{N}$ y $0^{\circ} 29,731^{\prime} \mathrm{E}$, en el municipio de Roquetes, comarca del Baix Ebre, provincia de Tarragona, a una altitud de $50 \mathrm{msnm}$. Ambos observatorios se encuentran separados por una distancia de 152 km en línea recta.

\section{Figura 4. Ubicación de los observatorios Fabra y del Ebro}

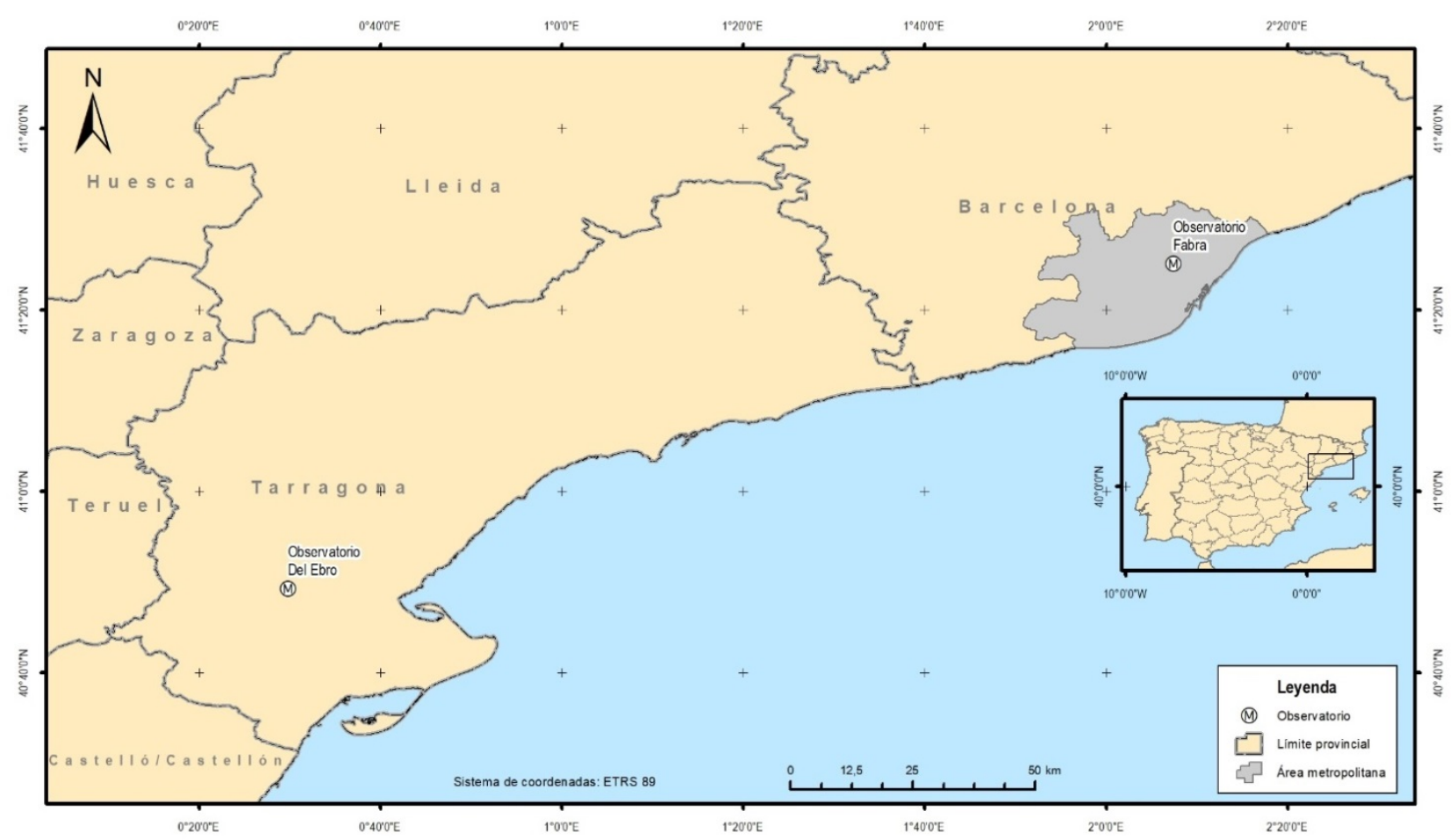

Fuente: elaboración propia

Para analizar las series climáticas de temperatura del aire en el área metropolitana de Barcelona, durante los años 1924 a 2016, se calculó el promedio anual de la temperatura media mensual para cada uno de los años de estudio, así como también se hallaron los promedios anuales de los valores medios máximos mensuales y mínimos mensuales registrados en el observatorio Fabra. Estos valores se representaron gráficamente para identificar la evolución interanual de las temperaturas máximas, medias y mínimas, con el objeto de determinar la tendencia de la temperatura a través de los años. Para ello, mediante una regresión lineal simple se estableció la relación de la temperatura con respecto al tiempo (años), siendo esta tendencia analizada estadísticamente para determinar su significación. Para realizar dicho análisis, se empleó el programa MAKESENS 1.0, desarrollado por el Instituto Meteorológico de Finlandia, el cual detecta y estima tendencias en series anuales de datos atmosféricos, basado en la prueba estadística de Mann-Kendall y la pendiente de Sen, que, respectivamente, determinan y estiman la tendencia monótona creciente o decreciente y la pendiente de una tendencial lineal (Salmi et al, 2002). Una 
vez hallada la ecuación de regresión, se calculó el incremento de la temperatura registrado en el observatorio Fabra durante los últimos 93 años.

Las series climáticas de temperatura media mensual (máxima, media y mínima) del observatorio del Ebro (observatorio de referencia), para el mismo periodo, fueron analizadas de la misma manera que los datos de Fabra. Cabe mencionar que, a fin de que las tendencias de ambos observatorios puedan ser comparadas, para hallar la ecuación de regresión de Fabra se optó por no considerar los datos de los 15 meses en los que el observatorio del Ebro presentó ausencia de información.

Se determinó la variación térmica entre ambos observatorios, para los valores máximos, medios y mínimos, mediante las diferencias entre el observatorio del Ebro y Fabra de 1924 a 2016, así como si la tendencia de esta variación, a través del programa MAKESENS 1.0, es significativa estadísticamente o no. Esta diferencia de temperaturas ha permitido establecer si el área metropolitana de Barcelona, debido a la presencia de la isla de calor urbano, ejerce influencia sobre la temperatura del aire en el observatorio Fabra. Dado que los observatorios del Ebro y Fabra se encuentran en la misma zona climática, es de esperar que la temperatura del aire se incremente en una tasa o variación casi similar por causa del cambio climático. Por ello, para obtener el aporte que ejerce el área metropolitana de Barcelona en el incremento de la temperatura del aire en Fabra, se compararon las tendencias de temperatura del observatorio Fabra y del observatorio del Ebro, para el subperiodo 1972 al 2016, de modo que el valor resultante de esta diferencia correspondería al incremento que registra Fabra por la influencia del calor urbano de Barcelona y su área metropolitana. Cabe indicar que se empleó solamente este subperiodo debido a que es a partir de los años 70 del siglo XX cuando se evidencia el incremento de la temperatura global por causa del cambio climático antropógeno.

\section{Resultados}

\subsection{Evolución temporal de la temperatura del aire}

La representación gráfica de la variación temporal de la temperatura media anual del aire (máxima, media y mínima) en el observatorio Fabra, desde el año 1924 al 2016, muestra el incremento de esta variable meteorológica en dicho lugar. De acuerdo a los valores anuales indicados en la Figura 5, se podrían establecer dos periodos con tendencias diferenciadas: (a) 1924-1971 y (b) 1972-2016. El primer periodo muestra valores de temperaturas variables, sin una clara tendencia, mientras que en el segundo periodo, a partir de 1972, se evidencia un incremento sostenido, que se manifiesta tanto para los valores anuales de las medias, como de las máximas y las mínimas. 
Figura 5. Evolución temporal de los promedios anuales de la temperatura media anual, máxima y mínima registrada en el observatorio Fabra, para el periodo 1924-2016

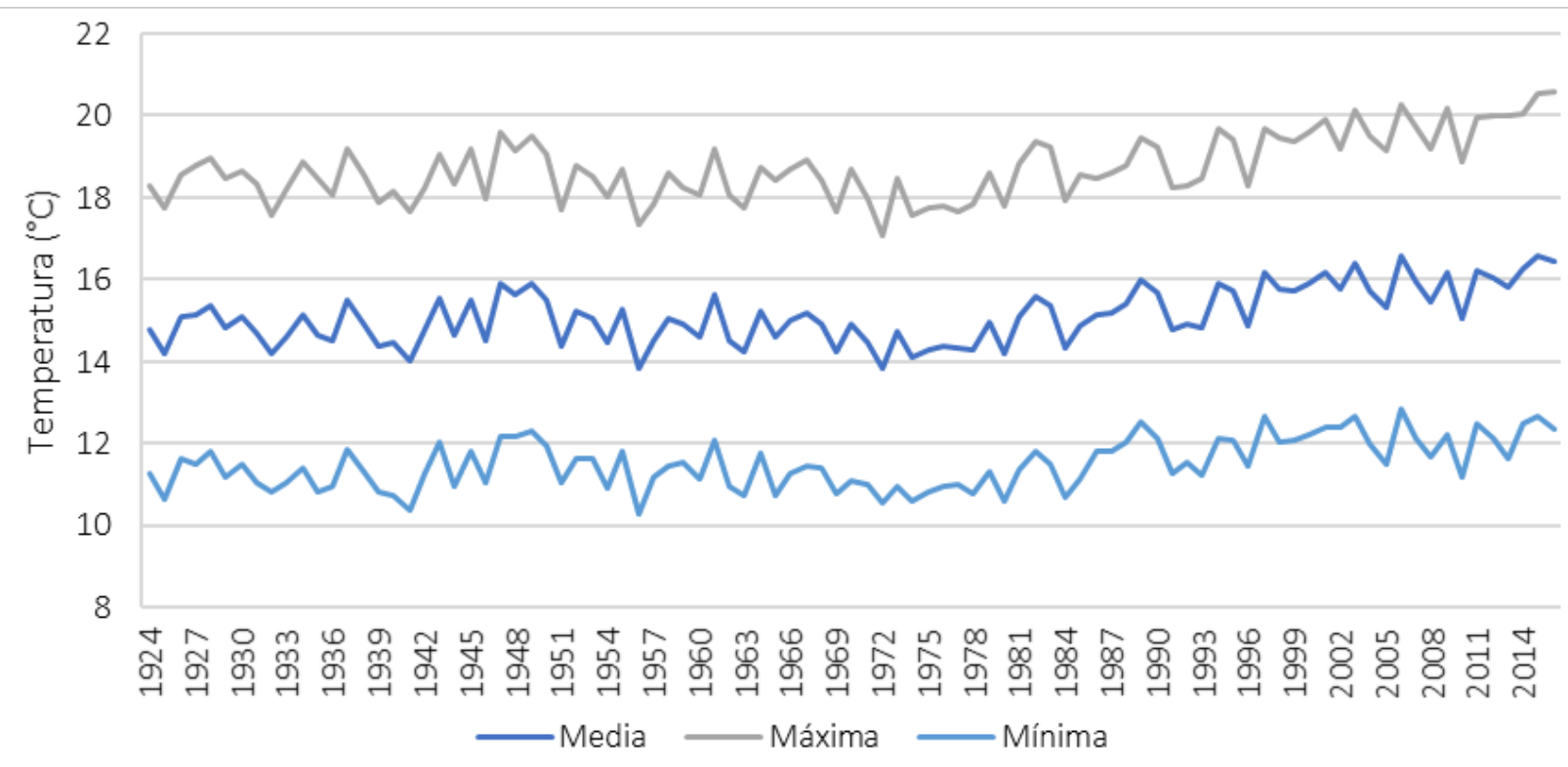

Fuente: elaboración propia

El valor máximo de la temperatura media anual de las máximas durante el periodo de evaluación fue de $20,6{ }^{\circ} \mathrm{C}$, en el año 2016 , mientras que el valor mínimo de la temperatura media anual de las mínimas fue de $10,3{ }^{\circ} \mathrm{C}$, en el año 1957 . Los valores máximos y mínimos de las temperaturas medias anuales máximas, medias y mínimas se presentan en la Tabla 2, así como los datos estadísticos básicos, tales como el promedio, la mediana y la desviación típica. Estos datos indican que la temperałura media anual de las máximas tiene una mayor dispersión, es decir que se ha registrado una mayor amplitud entre sus valores mínimos y máximos, así como evidencian que las tres variables de temperatura analizadas presentan una distribución similar a la normal, sin valores atípicos.

Tabla 2. Estadística básica de los promedios anuales de la temperatura media anual, máxima y mínima registrada en el observatorio Fabra, para el periodo 1924-2016 (en ${ }^{\circ} \mathrm{C}$ )

\begin{tabular}{|l|c|c|c|}
\hline Parámetros & $\begin{array}{c}\text { Temperatura media } \\
\text { anual de las máximas }\end{array}$ & $\begin{array}{c}\text { Temperatura media } \\
\text { anual de las medias }\end{array}$ & $\begin{array}{c}\text { Temperatura media } \\
\text { anual de las mínimas }\end{array}$ \\
\hline Valor mínimo & $17,1(1972)$ & $13,8(1972)$ & $10,3(1956)$ \\
\hline Valor máximo & $20,6(2016)$ & $16,6(2016)$ & $12,9(2006)$ \\
\hline Media & 18,7 & 15,1 & 11,5 \\
\hline Mediana & 18,6 & 15,1 & 11,5 \\
\hline Desviación estándar & 0,78 & 0,68 & 0,62 \\
\hline
\end{tabular}

Fuente: elaboración propia 
En el observatorio del Ebro también se aprecia el incremento de la temperatura del aire entre los años 1924 y 2016, tal como se muestra en la Figura 6. Sin embargo, a diferencia de Fabra, se puede observar un mayor incremento en los valores de la temperatura media anual de las máximas. Por otra parte, durante los 93 años analizados, se pueden establecer también dos periodos que presentan diferentes tendencias: (a) 1924-1971 y (b) 1972-2016, correspondiendo éstos a los mismos periodos identificados en el observatorio Fabra, aunque con una tasa de aumento mayor de la temperatura del aire en el último, especialmente en las máximas y mínimas.

Figura 6. Evolución temporal de los promedios anuales de la temperatura media anual, máxima y mínima registrada en el observatorio del Ebro, para el periodo 1924-2016

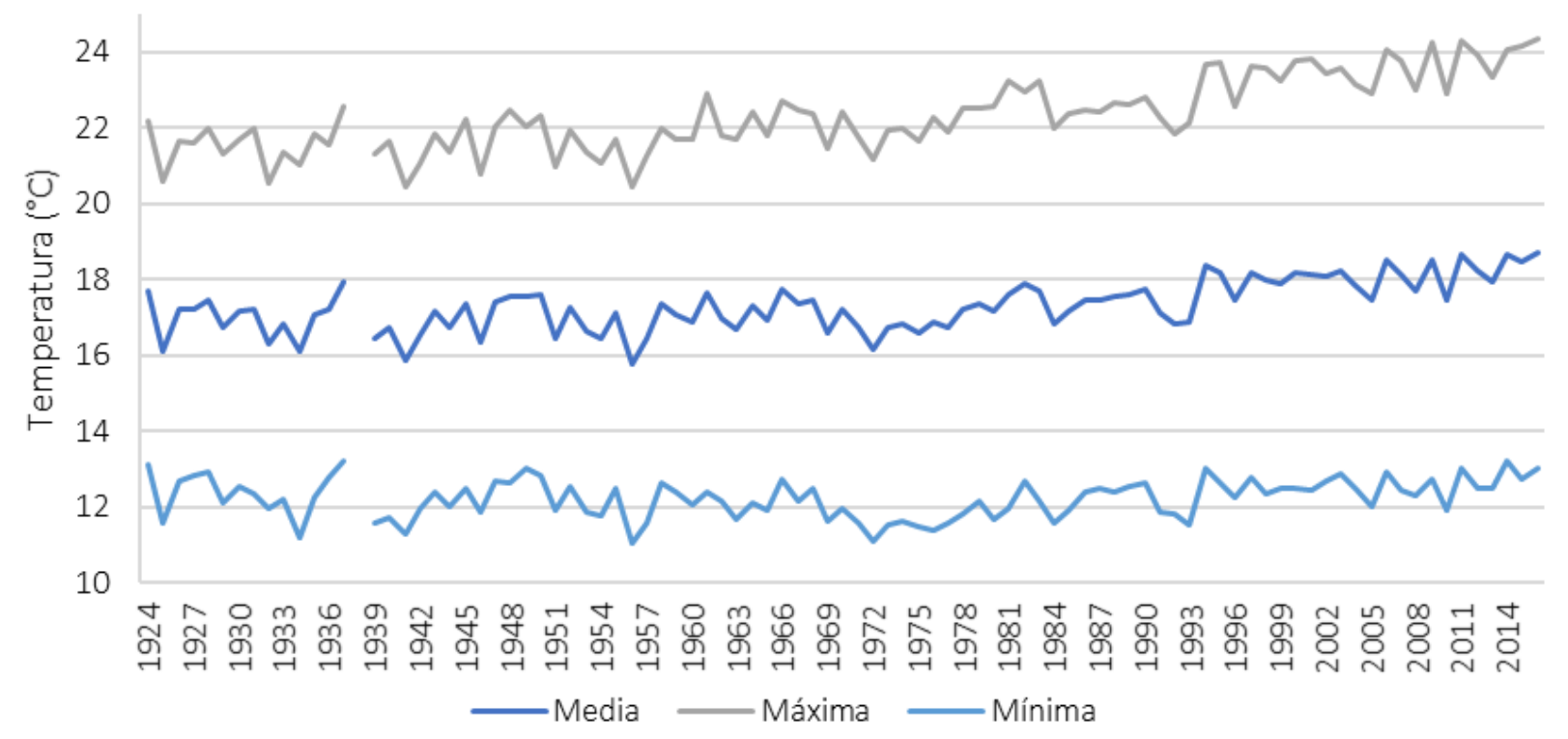

Fuente: elaboración propia

La máxima temperatura media anual de las máximas en el observatorio del Ebro se obtuvo en el año 2016 con un valor de $24,3{ }^{\circ} \mathrm{C}$, mientras que la mínima temperatura media anual de las mínimas fue de $11,0^{\circ} \mathrm{C}$ en el año 1956. La estadística básica de estos valores, indican que los datos tienden a la simetría y a una distribución similar a la normal, presentando los valores de la temperatura media anual de las máximas una mayor amplitud, tal como se aprecia en la Tabla 3.

La tendencia de las series históricas de las temperaturas media anuales y media anual de las máximas y de las mínimas se presentan en la Figura 7. Se aprecia que el observatorio Fabra ha registrado un incremento menor en las temperaturas medias y medias de las máximas, respecto a los valores del observatorio del Ebro, mientras que en las medias de las mínimas Fabra presentó un mayor incremento para los 93 años analizados. Asimismo, la Tabla 4 resume los datos de las pendientes de cada una de las ecuaciones de regresión lineal calculadas, así como el incremento que se ha evidenciado en los últimos 93 años. 
Tabla 3. Estadística básica de los promedios anuales de la temperatura media anual, máxima y mínima registrada en el observatorio del Ebro, para el periodo 1924-2016 (en ${ }^{\circ} \mathrm{C}$ )

\begin{tabular}{|l|c|c|c|}
\hline \multicolumn{1}{|c|}{ Parámetros } & $\begin{array}{c}\text { Temperatura media } \\
\text { anual de las máximas }\end{array}$ & $\begin{array}{c}\text { Temperatura media } \\
\text { anual de las medias }\end{array}$ & $\begin{array}{c}\text { Temperatura media } \\
\text { anual de las mínimas }\end{array}$ \\
\hline Valor mínimo & $20,4(1941)$ & $15,8(1956)$ & $11,0(1956)$ \\
\hline Valor máximo & $24,3(2016)$ & $18,7(2016)$ & $13,2(2014)$ \\
\hline Media & 22,3 & 17,3 & 12,3 \\
\hline Mediana & 22,3 & 17,3 & 12,4 \\
\hline Desviación estándar & 0,96 & 0,67 & 0,52 \\
\hline
\end{tabular}

Fuente: elaboración propia

Tabla 4. Tendencias de las temperaturas media anual y media anual de las máximas y de las mínimas de los observatorios Fabra y del Ebro en el período 1924-2016

\begin{tabular}{|l|c|c|c|c|}
\hline \multirow{2}{*}{$\begin{array}{l}\text { Temperatura media } \\
\text { anual }\end{array}$} & Pendiente de regresión lineal $\left({ }^{\circ} \mathrm{C} / \mathrm{año}\right)$ & \multicolumn{2}{c|}{ Incremento $\left(\right.$ 1924-2016) $\left({ }^{\circ} \mathrm{C}\right)$} \\
\cline { 2 - 5 } & Fabra & Ebro & Fabra & Ebro \\
\hline De las máximas & 0,0161 & 0,0289 & 1,50 & 2,69 \\
\hline Media & 0,0136 & 0,0160 & 1,26 & 1,49 \\
\hline De las mínimas & 0,0111 & 0,0032 & 1,03 & 0,30 \\
\hline
\end{tabular}

Fuente: elaboración propia

Mediante la aplicación del programa MAKESENS 1.0, se determinó la significación de las tendencias de las variables analizadas, resultando que los incrementos de las tres temperaturas en el observatorio Fabra son estadísticamente significativos, con un nivel de confianza del 99,9\%, al igual que los de las temperaturas medias y medias de las máximas del observatorio del Ebro, mientras que las medias de las mínimas presentan una tendencia que no es significativa.

De acuerdo a estos resultados, se concluye que la evolución temporal de la temperatura del aire en el observatorio Fabra durante los años 1924 y 2016, correspondiente a las máximas y las medias, presenta una tasa de incremento menor que la identificada en el observatorio del Ebro, que es el observatorio de referencia por ubicarse en una zona muy poco urbanizada y que no ha presentado cambios significativos en su entorno. Estos resultados no fueron los esperados, pues se consideraba que el observatorio Fabra presentaría una influencia térmica del calor urbano emitido por el área metropolitana de Barcelona, y de este modo el calentamiento del aire sería mayor que en el observatorio de referencia para el periodo 1924-2016. Sí que este resultado esperado se manifestó en los valores promedio anuales de las temperaturas mínimas, lo cual es consistente con el carácter nocturno del fenómeno de la isla de calor urbana (Moreno García, 2010). 
Figura 7. Rectas de regresión de las temperaturas media anual y media anual de las máximas y de las mínimas de los observatorios Fabra y del Ebro, para el periodo 1924-2016

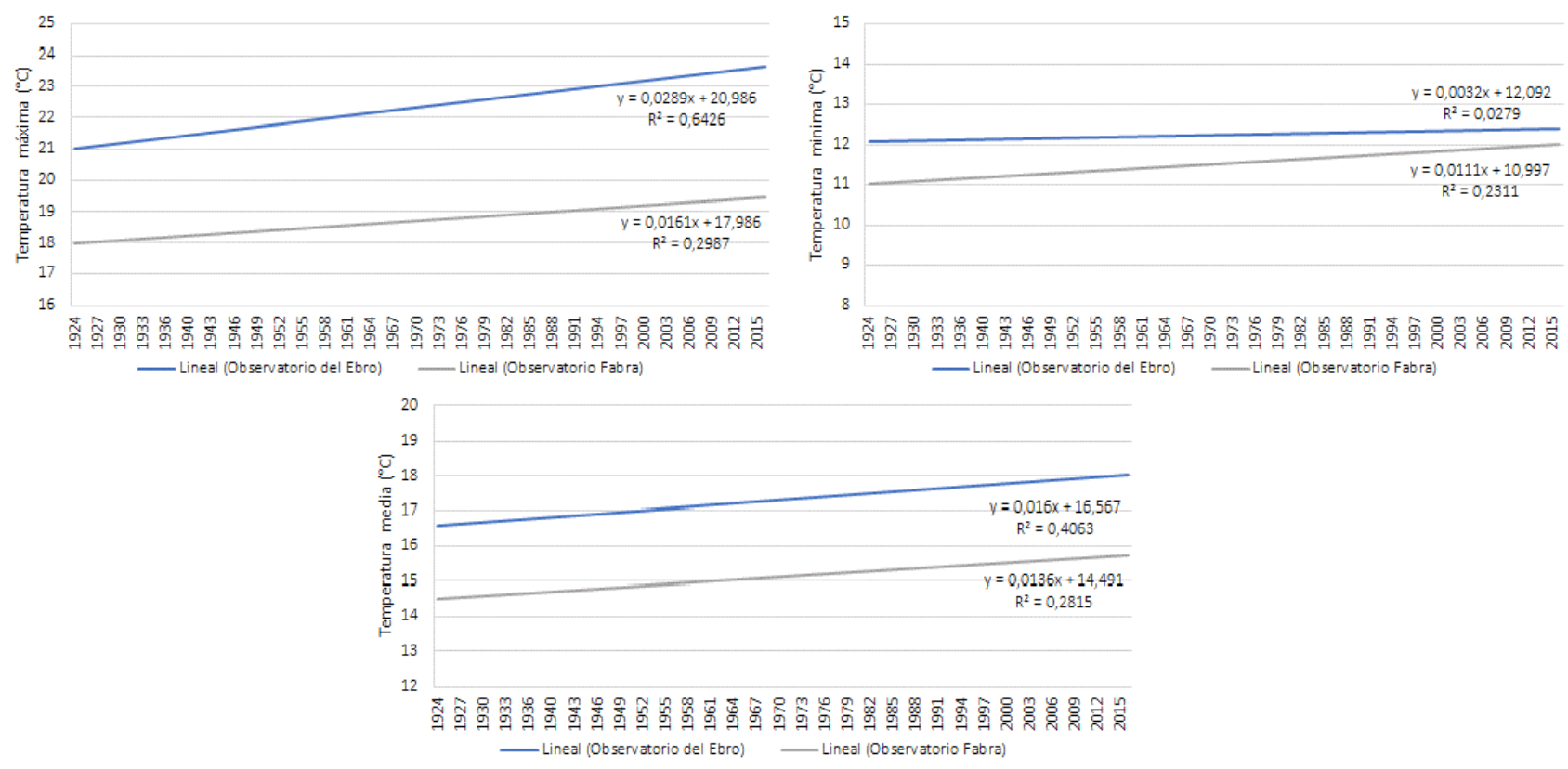

Fuente: elaboración propia

Figura 8. Evolución temporal de las temperaturas media anual y media anual de las máximas y de las mínimas en los observatorios Fabra y del Ebro,

para los subperiodos 1924-1971 y 1972-2016

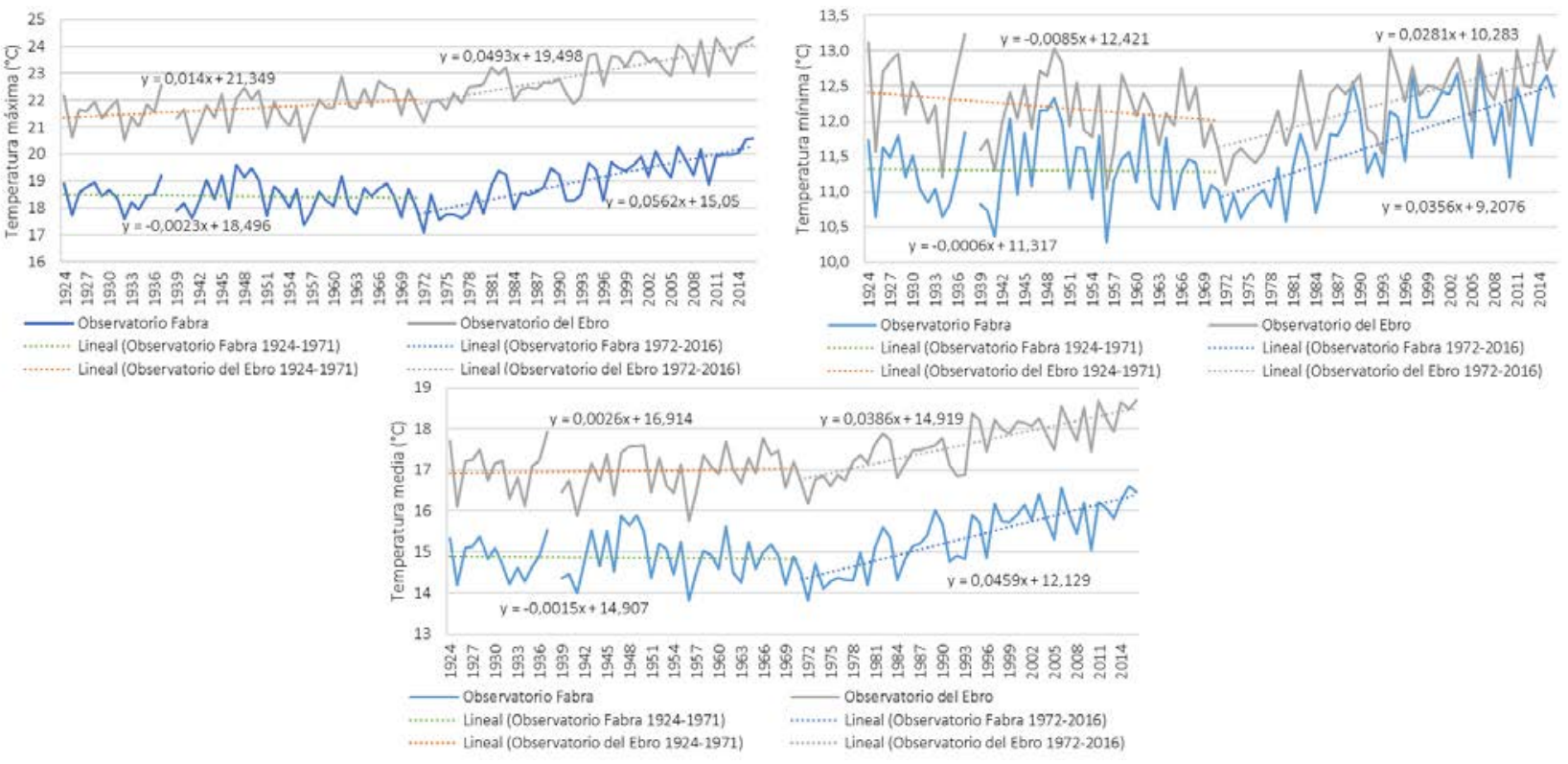

Fuente: elaboración propia 
Por otra parte, al analizar las tendencias para los subperiodos de 1924 a 1971 y de 1972 a 2016, se observa que Fabra, a partir del año 1972, presenta un incremento más acelerado de la temperatura que el observatorio del Ebro, para las tres temperaturas analizadas, tal como se aprecia en la Figura 8.

El análisis de la significación, a través del programa MAKESENS 1.0, indica que en Fabra los incrementos de las tres temperaturas (media, media de las máximas y media de las mínimas) para el subperiodo 1972 a 2016 es significativa, con un nivel de confianza del 99,9\%, mientras que las tendencias para el subperiodo 1924 a 1971 no presentan significación estadística. En el caso del observatorio del Ebro, para el subperiodo 1971-2016, se evidenció también un aumento significativo de las tres temperaturas analizadas con un nivel de confianza del 99,9\%, mientras que la evidencia estadística para el subperiodo 1924-1971, señala que hay una tendencia positiva significativa con un nivel de confianza del 95,0\% para la media de las máximas, una tendencia negativa con un nivel de confianza del 90,0\% para la media de las mínimas y que no se registra tendencia en los valores de temperatura media.

En la Tabla 5 se presenta un resumen de las pendientes de la recta de regresión lineal de las tendencias de las tres temperaturas para cada subperiodo.

Tabla 5. Tendencias de las temperaturas media anuales y media anual de las máximas y de las mínimas de los observatorios Fabra y del Ebro, para los subperiodos 1924-1971 y 1972-2016

\begin{tabular}{|c|c|c|c|c|c|}
\hline \multirow{2}{*}{ Subperiodo } & \multirow{2}{*}{$\begin{array}{c}\text { Temperatura } \\
\text { media anual }\end{array}$} & \multicolumn{2}{|c|}{$\begin{array}{c}\text { Pendiente de regresión lineal } \\
\left({ }^{\circ} \mathrm{C} / \text { año) }\right.\end{array}$} & \multicolumn{2}{c|}{ Incremento $\left({ }^{\circ} \mathrm{C}\right)$} \\
\cline { 3 - 6 } & & Fabra & Ebro & Fabra & Ebro \\
\hline \multirow{3}{*}{$1924-1971$} & De las máximas & $-0,0023$ & 0,014 & $-0,11$ & 0,67 \\
\cline { 2 - 6 } & Media & $-0,0015$ & 0,0026 & $-0,07$ & 0,12 \\
\cline { 2 - 6 } & De las mínimas & $-0,0006$ & $-0,0085$ & $-0,03$ & $-0,40$ \\
\hline \multirow{3}{*}{$1972-2016$} & De las máximas & 0,0562 & 0,0493 & 2,53 & 2,21 \\
\cline { 2 - 6 } & Media & 0,0459 & 0,0386 & 2,07 & 1,74 \\
\cline { 2 - 6 } & De las mínimas & 0,0356 & 0,0281 & 1,60 & 1,26 \\
\hline
\end{tabular}

Fuente: elaboración propia

El incremento de las temperaturas en los dos observatorios analizados muestra la misma tendencia que registran los datos globales y los registrados en España (Grupo Intergubernamental de Expertos sobre el Cambio Climático, 2015; Castro, Martín Vide, \& Alonso, 2005), es decir que a partir de los años 70 el incremento de la temperatura de aire se hace más evidente. Este incremento se aprecia en los datos obtenidos en los observatorios Fabra y del Ebro por lo que en ambos se evidencia, para el subperiodo 1972-2016, la señal correspondiente al calentamiento global por 
origen antropógeno. Sin embargo, la mayor tasa de incremento de las temperaturas en Fabra refleja la señal del calentamiento global y apunta a un plus térmico urbano.

Adicionalmente, también se representó la evolución temporal de las diferencias de temperaturas entre el observatorio Fabra y el observatorio de referencia para identificar si existe una tendencia al incremento de diferencias entre ambos observatorios. Así como también, se analizó las tendencias de los dos subperiodos, observándose que a partir del año 1972 hay un desaceleración y cambio de tendencia de las diferencias térmicas de las temperaturas media anual de las máximas y media entre el observatorio de referencia y Fabra.

Figura 9. Rectas de regresión de las diferencias entre las temperaturas media anual y media anual de las máximas y de las mínimas de los observatorios del Ebro y Fabra, para el periodo 1924-2016 y para los subperiodos 1924-1971 y 1972-2016

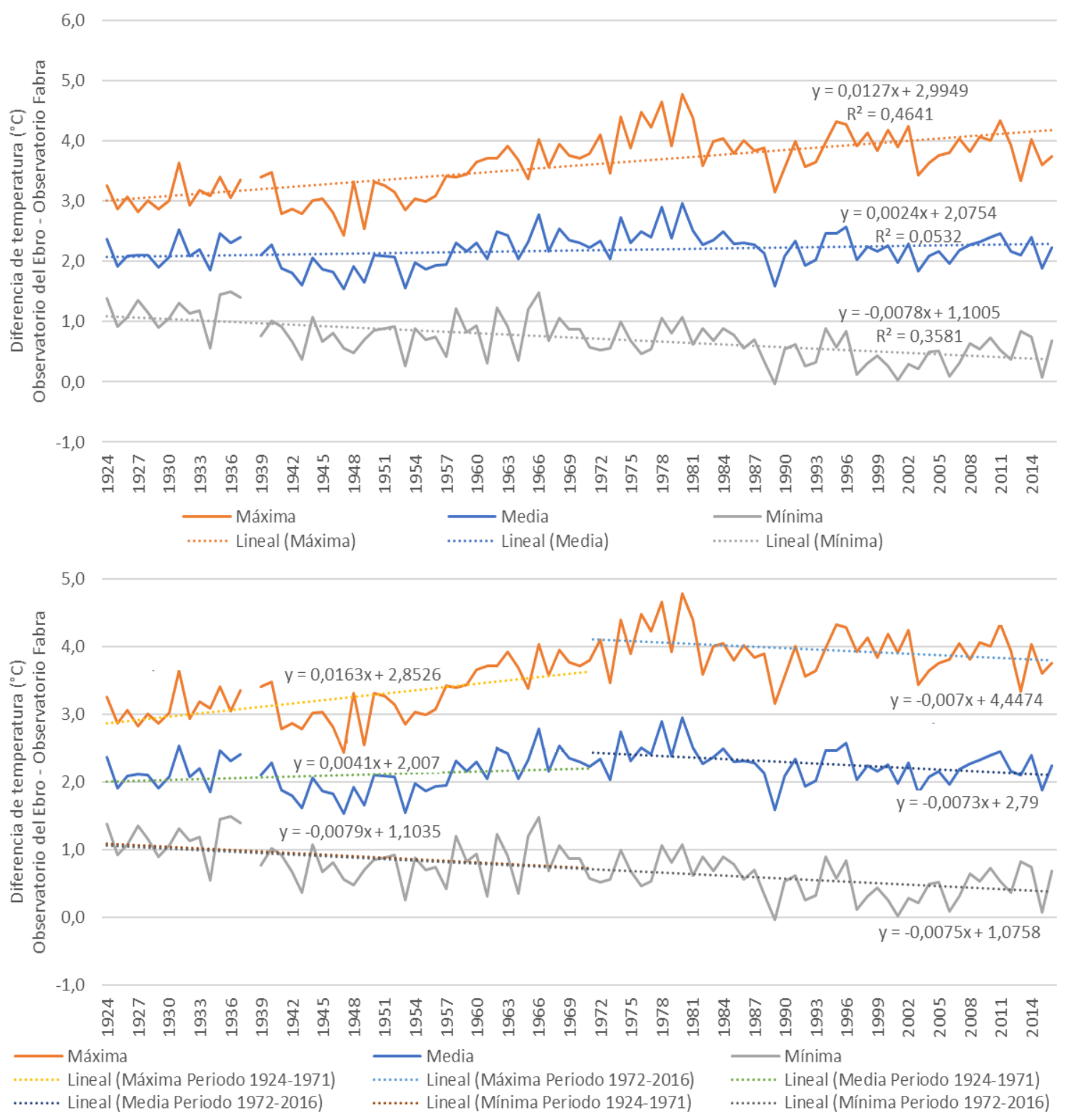

Fuente: elaboración propia 
Por otra parte, se evaluó la significación de la diferencia de las temperaturas entre el observatorio del Ebro y el observatorio Fabra para determinar si la tendencia es significativa. Los resultados indican que, para el periodo analizado, las diferencias de los valores de las temperaturas medias anuales de las máximas y de las mínimas presentan una tendencia significativa con un nivel de confianza del 99,9\%, mientras que la tendencia de la temperatura media anual presenta una significación del $95 \%$, tal como se presenta en la Tabla 6.

Tabla 6. Tendencias de las diferencias entre las temperaturas media anual y media anual de las máximas y de las mínimas de los observatorios del Ebro y Fabra, para el periodo 1924-2016 y para los subperiodos 1924-1971 y 1972-2016

\begin{tabular}{|c|c|c|c|}
\hline Periodo & $\begin{array}{l}\text { Diferencia entre las } \\
\text { temperaturas }\end{array}$ & $\begin{array}{c}\text { Pendiente de regresión lineal } \\
\text { (en }{ }^{\circ} \mathrm{C} / \text { año) }\end{array}$ & Nivel de significación \\
\hline \multirow{3}{*}{ 1924-2016 } & $\begin{array}{l}\text { Media de las } \\
\text { máximas }\end{array}$ & 0,0127 & $99,9 \%$ \\
\hline & Media & 0,0024 & $95,0 \%$ \\
\hline & $\begin{array}{l}\text { Media de las } \\
\text { mínimas }\end{array}$ & $-0,0078$ & $99,9 \%$ \\
\hline \multirow{3}{*}{ 1924-1971 } & $\begin{array}{l}\text { Media de las } \\
\text { máximas }\end{array}$ & 0,0163 & $99,9 \%$ \\
\hline & Media & 0,0041 & \\
\hline & $\begin{array}{l}\text { Media de las } \\
\text { mínimas }\end{array}$ & $-0,0079$ & $95,0 \%$ \\
\hline \multirow{3}{*}{ 1972-2016 } & $\begin{array}{l}\text { Media de las } \\
\text { máximas }\end{array}$ & $-0,0070$ & \\
\hline & Media & $-0,0073$ & $95,0 \%$ \\
\hline & $\begin{array}{l}\text { Media de las } \\
\text { mínimas }\end{array}$ & $-0,0075$ & $95,0 \%$ \\
\hline
\end{tabular}

Fuente: elaboración propia

De la tabla anterior, se aprecia que para el subperiodo 1924-1971 la tendencia de las diferencias térmicas de los valores anuales de la temperatura media de las máximas entre el observatorio del Ebro y Fabra es significativa hacia el incremento, es decir que el observatorio de referencia ha aumentado su temperatura con mayor velocidad que el de Fabra, mientras que para el segundo subperiodo se presenta la situación inversa registrándose una tendencia negativa que no es significativa. Con respecto a la variación térmica de los valores anuales de la temperatura media, se aprecia que no hay una tendencia significativa a favor del observatorio del Ebro para el subperiodo del 1924 a 1971, mientras que, a partir de 1972, se aprecia que Fabra tiende a presentar un ligero incremento de temperatura en comparación con el observatorio de referencia, siendo significativo estadísticamente con un nivel de confianza del 95,0\%. Finalmente, con respecto a las diferencias térmicas de los valores de la temperatura media de las mínimas de ambos observatorios se aprecia que, para los dos subperiodos, la tendencia es negativa y significativa al 95,0\% de nivel de confianza, es decir, que la diferencia térmica entre el observatorio del Ebro y el observatorio Fabra, 
conforme transcurren los años, aumenta a favor del segundo. Sin embargo, cabe indicar que se evidencia una muy ligera atenuación de este incremento en el subperiodo de 1972 a 2016, con respecto al de 1924 a 1971.

\subsection{Aporte de calor urbano en la temperatura del aire}

La temperatura media anual del aire en el observatorio de Fabra aumentó en $1,26^{\circ} \mathrm{C}$ en el periodo 1924 a 2016, mientras que el observatorio de referencia presentó un incremento de 1,49 ${ }^{\circ} \mathrm{C}$. Sin embargo, considerando solo el subperiodo de 1972 a 2016, que coincide con los años donde se evidencia el incremento sostenido de la temperatura del aire a nivel mundial asociándolo al cambio climático, el incremento de la temperatura media anual en Fabra es de $2,07^{\circ} \mathrm{C}$ mientras que en el observatorio del Ebro es de $1,74{ }^{\circ} \mathrm{C}$. Asimismo, considerando la temperatura media de las máximas y en la media de las mínimas, se registraron respectivamente un incremento de $1,60{ }^{\circ} \mathrm{C}$ y $2,53^{\circ} \mathrm{C}$ en Fabra, y de $1,26{ }^{\circ} \mathrm{C}$ y $2,21^{\circ} \mathrm{C}$ en el observatorio del Ebro.

Dado que el observatorio del Ebro se encuentra en un área con una baja urbanización (municipio de Roquetes), que podría considerarse como rural, y considerando que dicha área no ha sufrido modificaciones significativas en sus características físicas, el incremento de la temperatura del aire registrado en él puede atribuirse principalmente al calentamiento global. Por lo tanto, si a la tasa de incremento de temperatura media, en el subperíodo 1972 a 2016, del observatorio Fabra $\left(0,0459^{\circ} \mathrm{C} / \mathrm{año}\right)$ se le resta el valor de la tasa correspondiente al observatorio de referencia $\left(0,0386^{\circ} \mathrm{C} / \mathrm{año}\right)$, la diferencia puede considerarse debida al efecto del clima urbano local. Esa diferencia es de $0,0073^{\circ} \mathrm{C}$ por año, tal como se aprecia en la Tabla 7. Este resultado indica que el área metropolitana de Barcelona contribuiría si no cambiara la ciudad, cada 100 años, en $0,73^{\circ} \mathrm{C}$ en el calentamiento del aire registrado en el observatorio de Fabra.

Tabla 7. Diferencia de las tasas de incremento de las temperaturas media anual y media anual de las máximas y de las mínimas entre los observatorios Fabra y del Ebro,

para el subperiodo 1972-2016

\begin{tabular}{|c|c|c|c|c|}
\hline \multirow[b]{2}{*}{ Temperatura } & \multicolumn{2}{|c|}{ Pendiente de regresión ( $\left.{ }^{\circ} \mathrm{C} / \mathrm{año}\right)$} & \multirow{2}{*}{$\begin{array}{l}\text { Diferencia de pendientes } \\
\qquad\left({ }^{\circ} \mathrm{C} / \text { año) }\right.\end{array}$} & \multirow{2}{*}{$\begin{array}{l}\text { Diferencia de } \\
\text { pendientes } \\
\left({ }^{\circ} \mathrm{C} / \text { siglo) }\right.\end{array}$} \\
\hline & Observatorio Fabra & $\begin{array}{c}\text { Observatorio del } \\
\text { Ebro }\end{array}$ & & \\
\hline $\begin{array}{l}\text { Media de las } \\
\text { máximas }\end{array}$ & 0,0562 & 0,0493 & 0,0069 & 0,69 \\
\hline Media & 0,0459 & 0,0386 & 0,0073 & 0,73 \\
\hline $\begin{array}{l}\text { Media de las } \\
\text { mínimas }\end{array}$ & 0,0356 & 0,0281 & 0,0075 & 0,75 \\
\hline
\end{tabular}

Fuente: elaboración propia

Este aporte o liberación de calor urbano, a escala local, se percibe con mayor intensidad en el incremento de la temperatura del aire en horas nocturnas (temperatura media de las mínimas) en 
Barcelona y su área metropolitana, con un valor de $0,0075{ }^{\circ} \mathrm{C}$ más en Fabra que en observatorio del Ebro, observatorio de referencia, es decir, un aporte equivalente a $0,75^{\circ} \mathrm{C}$ de incremento por siglo, calculado sobre el subperíodo 1972 a 2016. Este aporte local de la ciudad en la temperatura es apreciablemente inferior al que produce el calentamiento global. Hay que añadir aquí que, por la ubicación litoral del área metropolitana de Barcelona, esta se beneficia del efecto termorregulador del mar, lo que pudiera amortiguar la señal térmica local. Resumiendo, la señal térmica local en la temperatura media de las mínimas, variable en la que se evidencia más claramente la influencia del área metropolitana de Barcelona en el incremento de la temperatura del aire, tiene un peso de un $21 \%$, y la señal térmica global (calentamiento global) contribuye a incrementar la temperatura media de las mínimas en un $79 \%$, en el observatorio Fabra. ${ }^{6}$

\section{Conclusiones}

El incremento de la temperatura del aire (media de las máximas, media y media de las mínimas) en el observatorio Fabra es significativo para el periodo 1924 a 2016, presentando un aumento de $1,50{ }^{\circ} \mathrm{C}$ para la temperatura media de las máximas, $1,26{ }^{\circ} \mathrm{C}$ para la temperatura media y $1,03{ }^{\circ} \mathrm{C}$ para la temperatura media de las mínimas. Estos resultados indican que el aire del área metropolitana de Barcelona se está calentando, mostrando la misma tendencia hallada en los estudios relacionados al cambio climático a nivel global (Grupo Intergubernamental de Expertos sobre el Cambio Climático, 2015), así como a otros análisis para Barcelona (Institut d’Estudis Catalans y Generalitat de Catalunya, 2016).

El observatorio Fabra presenta una mayor tasa de incremento de temperatura para el subperiodo 1972-2016 que para el subperiodo 1924-1971, confirmando lo señalado por el IPCC (2015), en el que se indica que a partir de los años 70 del siglo XX se evidencia una aceleración del calentamiento global asociado a las actividades humanas. Por ello, se puede afirmar que una parte sustancial del incremento de temperatura registrado en el observatorio Fabra durante 1972-2016 se debe principalmente al cambio climático.

Durante este subperiodo (1972-2016) se ha identificado que el incremento de la temperatura del aire en Fabra es superior al registrado en el observatorio del Ebro. Este último observatorio, al ubicarse en un área no urbana y no haber presentado variaciones significativas en su entorno y morfología, registra valores de temperatura que reflejan la influencia global, sin una señal térmica local urbana. De todo ello, puede concluirse que la temperatura registrada en el observatorio Fabra presenta una influencia del área metropolitana de Barcelona, la cual aporta calor. Sumado al

6 El cálculo de estos valores se realizó a partir de los datos de pendiente de regresión ( ${ }^{\circ} \mathrm{C} /$ año) del subperiodo 1972-2016, representando el observatorio Fabra el $100 \%$. 
calentamiento global, origina que la tasa de incremento de temperatura en Fabra sea más alta que en el observatorio del Ebro.

La principal causa del incremento de la temperatura del aire en el observatorio Fabra es el calentamiento global. Sin embargo, los resultados han evidenciado que el área metropolitana de Barcelona también contribuye, en menor medida, en el incremento de la temperatura del aire, por lo que en Barcelona se puede distinguir dos tipos de influencia térmica: la señal térmica global expresada a través del calentamiento global y la señal térmica local, que correspondería al calor urbano atrapado y generado por la ciudad. Los resultados indican que en los registros medios anuales de la temperatura media de las mínimas se aprecia un mayor aporte de calor urbano, que en la temperatura media y en la media de las máximas: $0,69^{\circ} \mathrm{C} /$ siglo para la temperatura media de las máximas, $0,73^{\circ} \mathrm{C} /$ siglo para la media y $0,75^{\circ} \mathrm{C}$ para la media de las mínimas (valores calculados sobre el subperíodo 1972 a 2016).

Aunque el calor urbano aportado por el área metropolitana de Barcelona es inferior (un 21,0 \% en la serie de temperatura media de las mínimas) al derivado del calentamiento global, se debe considerar este impacto en los instrumentos de planificación territorial, tanto a nivel metropolitano como a nivel municipal, para intentar reducirlo. La planificación del área metropolitana de Barcelona debe considerar el efecto de la isla de calor urbana de manera que se controlen los factores que originan la retención y producción de calor urbano. Durante las olas de calor las temperaturas mínimas muy elevadas en el centro de Barcelona, por el plus térmico de la isla de calor, aumentan significativamente la morbilidad y la mortalidad de ancianos y personas con enfermedades crónicas. En este sentido, la isla de calor barcelonesa es hoy un riesgo meteorológico. Se trata, pues, de reducir la intensidad de la isla de calor para mejorar este aspecto de salud pública, que conllevará la mejora del confort térmico de los barceloneses durante los meses de verano.

Agradecimientos: A Marc Prohom i Duran del Servei Meteorològic de Catalunya por brindar con generosidad la información meteorológica empleada en el presente artículo.

Declaración responsable: Las/os autoras/es declaran que no existe ningún conflicto de interés en relación a la publicación de este artículo. Patricia Barros ha desarrollado el marco teórico, el procesamiento y análisis de los resultados bajo la asesoría constante de Javier Martin-Vide. 


\section{Bibliografía}

Alcañiz, M. (2008). Cambios demográficos en la sociedad global. Papeles de Población, 14(57), 227-255. Retrieved from http://www.scielo.org.mx/scielo.php?script=sci_arttext\&pid=S1405$\underline{74252008000300011}$

Àrea Metropolitana de Barcelona \& Barcelona Regional. (2014). Pla de Sostenibilitat Ambiental de L'Àrea Metropolitana de Barcelona 2014-2020. Barcelona: Àrea Metropolitana de Barcelona. Retrieved from http://www.amb.cat/ca/web/medi-ambient/actualitat/publicacions/detall//publicacio/memoria-del-psamb/1138518/11818

Castro, M. de, Martín Vide, J., \& Alonso, S. (2005). El clima de España: Pasado, presente y escenarios de clima para el siglo XXI. Evaluación Preliminar de los impactos en España por efecto del cambio climático. Madrid: Ministerio de Medio Ambiente. Retrieved from https://digital.csic.es/handle/10261/35782

Emmanuel, R., \& Krüger, E. (2012). Urban heat island and its impact on climate change resilience in a shrinking city: The case of Glasgow, UK. Building and Environment, 53, 137-149.

Estrada, F., Botzen, W. J. W., \& Tol, R. S. J. (2017). A global economic assessment of city policy to reduce climate change impacts. Nature Climate Change, 7, 403406. http://dx.doi.org/10.1038/NCLIMATE3301

Ferrer i Aixalà, A. (1996). Els polígons de Barcelona. Barcelona: Edicions de la Universitat politecnica de Catalunya.

Fondo de Población de las Naciones Unidas. (2016). Estado de la población mundial 2016 (edited by R. Kollodge). New York: Fondo de Población de las Naciones Unidas. Retrieved from http://www.unfpa.org/sites/default/files/sowp/downloads/The_State_of_World_Population 2016_-_Spanish.pdf

Grimm, N. B., Faeth, S. H., Golubiewski, N. E., Redman, C. L., Wu, J., Bai, X., \& Briggs, J. M. (2008). Global Change and the Ecology of Cities. Science, 379, 756. http://dx.doi.org/10.1126/science. 1150195

Grupo intergubernamental de expertos sobre el cambio climático (2015). Cambio climático 2014: Informe de síntesis (R. K. Pachauri \& L. Meyer, Eds.). Geneve: IPCC. Retrieved from http://www.ipcc.ch/pdf/assessment-report/ar5/syr/SYR_AR5_FINAL_full_es.pdf

Hamdi, R., Deckmyn, A., Termonia, P., Demarée, J. A., Baguis, P., Vanhuysse, S., \& Wolff, E. (2009). Effects of historical urbanization in the Brussels Capital Region on surface air temperature time series: a model study. Journal of Applied Meteorology and Climatology, 48, 2181-2196. 
Hamdi, R., Giot, O., de Troch, R., Deckmyn, A., \& Termonia, P. (2015). Future climate of Brussels and Paris for the 2050 s under the A1B scenario. Urban climate, 12 . 160182. http://dx.doi.org/10.1016/j.uclim.2015.03.003

Hamdi, R., \& van de Vyver, H. (2011). Estimating urban heat island effects on near-surface air temperature records of Uccle (Brussels, Belgium): An observational and modeling study. Advances in Sciences and Research, 6, 27-34.

Henry, J. G., \& Heinke, G. W. (1999). Ingenieria ambiental (2nd ed.). México D.F.: Prentice-Hall Hispanoamericana.

Hwang, R-L., Lin, C-Y, \& Huang, K-T. (2017). Spatial and temporal analysis of urban heat island and global warming on residential thermal comfort and cooling energy in Taiwan. Energy and Buildings, 152(1), 804-812.

Institut d'estadística De Catalunya (2016, June 27). Padrón municipal de habitantes. Padró municipal d'habitants. Xifres oficials. Per sexe. Municipis. In Idescat. Retrieved from https: / $/$ www.idescat.cat/pub/?id=pmh\&n=446\&lang=es\&by=mun\#Plegable=geo

Institut d'estudis Catalans, \& Generalitat de Catalunya. (2016). Tercer Informe Sobre el Canvi Climàtic a Catalunya. Barcelona: Institut d'Estudis Catalans y Generalitat de Catalunya. Retrieved from http://cads.gencat.cat/ca/detalls/detallarticle/Tercer-informe-sobre-el-canvi-climatic-aCatalunya-00003

Jones, P. D., \& Lister, D. H. (2009). The urban heat island in central London and urban-related warming trends in Central London since 1900. Weather, 64, 323-327.

Martín Vide, J. (2008). La nueva realidad del calentamiento global. Un decálogo del cambio climático. Scripta Nova, XII, 270(23). Retrieved from http://www.ub.edu/geocrit/sn/sn-270/sn$\underline{270-23 . h t m}$

Martín Vide, J. (2016). Evolució recent de la temperatura, la precipitació i altres variables climàtiques a Catalunya. In Tercer informe sobre el canvi climàtic a Catalunya (pp. 93-112). Barcelona: Institut d'Estudis Catalans y Generalitat de Catalunya. Retrieved from hittp://cads.gencat.cat/ca/detalls/detallarticle/Tercer-informe-sobre-el-canvi-climatic-aCatalunya-00003

Moreno García, M. del C. (2010). Climatologia urbana (2nd ed.). Barcelona: Publicacions i Edicions, Universitat de Barcelona.

Moreno Rodríguez, J. M. (2005). Principales conclusiones de la evaluación preliminar de los impactos en España por efecto del cambio climático (vol. 1). Madrid: Oficina Española de Cambio Climático. Retrieved from www.oei.es/historico/decada/portadas/impactos_Espanha.pdf 
Organización de las Naciones Unidas (2016, June 21). Población urbana (\% del total). Data. Retrieved from http://datos.bancomundial.org/indicator/SP.URB.TOTL.IN.ZS

Sachindra, D. A., Ng, A. W. M., Muthukumaran, S., \& Perera, B. J. C. (2016). Impact of climate change on urban heat island effect and extreme temperatures: a case-study. Quarterly Journal of the Royal Meteorological Society, 142(694), 172-186. http://dx.doi.org/10.1002/aj.2642

Salmi, T., Määttä, A., Anttila, P., Ruoho-Airola, T., \& Amnell, T. (2002). Detecting Trends of Annual Values of Atmospheric Pollutants by the Mann-Kendall Test and Sen's Slope Estimates -The Excel Template Application Makesens. Publications on Air Quality, 37(35). Retrieved from http://www.ilmanlaatu.fi/ilmansaasteet/julkaisu/pdf/MAKESENS-Manual_2002.pdf

U.S. EPA (2008). Urban Heat Island Basics. In Reducing Urban Heat Islands: Compendium of Strategies (p. 22). Washington D.C.: U.S Environmental Protection Agency. Retrieved from www.epa.gov/heatisland/resources/pdf/BasicsCompendium.pdf

World Meteorological Organization (n.d.). Commission for Climatology. Frequently Asked Questions (FAQs). Retrieved from http://www.wmo.int/pages/prog/wcp/ccl/fags. php 